\title{
Evolução Clínica de Pacientes com Lesões de Tronco de Coronária Esquerda Não-Protegido Submetidos a Angioplastia Coronária com Implante de Stents Farmacológicos
}

\author{
Costantino R. Costantini ${ }^{1}$, Daniel Zanuttini ${ }^{1}$, Marcos A. Denk ${ }^{1}$, Sergio G. Tarbine ${ }^{1}$, Marcelo F. Santos ${ }^{1}$, \\ Eduardo F. Oliveira' ${ }^{1}$, Marcos H. Bubna', José F. Rocha ${ }^{1}$, Marcos J. Barbosa' ${ }^{1}$, Costantino O. Costantini ${ }^{1}$
}

\section{RESUMO}

Introdução: Recentemente a intervenção coronária percutânea (ICP) com stents farmacológicos (SFs) tem se mostrado uma opção viável em pacientes selecionados com lesão de tronco de coronária esquerda não-protegido (TCE-NP). Este estudo teve como objetivo avaliar a efetividade e a segurança da ICP com SFs em lesões de TCE-NP da prática diária, analisando a ocorrência combinada de eventos cardíacos adversos maiores (ECAM) a longo prazo. Métodos: Foram tratados 142 pacientes consecutivos, com média de seguimento clínico de 917 + 743 dias. A decisão de utilizar um ou dois stents e inibidor da glicoproteína Ilb/Illa ficou a critério do operador. Angiografia coronária no seguimento não foi realizada de rotina, mas deixada a critério clínico. Resultados: A média de idade foi de $67,5 \pm 16$ anos, três quartos dos pacientes eram do sexo masculino, 29\% eram portadores de diabetes e $39 \%$ apresentavam angina instável. Foram utilizados $2,75 \pm 1,25$ stents por paciente. Lesões com comprometimento da bifurcação foram identificadas em $90,1 \%$ e as técnicas mais frequentemente utilizadas foram o provisional stent em $36 \%$ e o small crush em $29 \%$ dos pacientes. Ultrassom intracoronário foi realizado em $92,3 \%$ dos pacientes, e reintervenção ocorreu em 21,3\% dos stents, por apresentarem aposição incompleta de suas hastes após o implante. ECAM na evolução tardia ocorreram em 15,4\%, óbito cardíaco ocorreu em 3,6\%, revascularização do vaso-alvo em $11,2 \%$ e trombose definitiva/ provável do stent em 1,4\%. Conclusões: A ICP com SFs em lesões de TCE-NP neste estudo mostrou ser segura e eficaz na evolução tardia, com baixas taxas de óbito cardíaco e de trombose do stent.

DESCRITORES: Doença da artéria coronária. Angioplastia. Stents farmacológicos.

\author{
ABSTRACT \\ Clinical Follow-up of Patients with Unprotected \\ Left Main Disease Undergoing Percutaneous \\ Coronary Intervention with Drug-Eluting Stents
}

Background: Recently, percutaneous coronary intervention (PCI) with drug-eluting stents (DES) has proven to be a feasible option in selected patients with unprotected left main (LM) disease. This study was aimed at assessing the efficacy and safety of $\mathrm{PCl}$ with DES in LM lesions in the daily practice, analyzing the long-term occurrence of major adverse cardiac events (MACE). Methods: A total of 142 consecutive patients were treated with a mean follow-up of $917 \pm 743$ days. The decision to use one or two stents or glycoprotein $\mathrm{Ilb} / \mathrm{Illa}$ inhibitors was left to the operator's discretion. Coronary angiography was not performed routinely in the follow-up. Results: Mean age was $67.5 \pm 16$ years, $75 \%$ of the patients were male, $29 \%$ had diabetes and $39 \%$ had unstable angina. A total of $2.75+1.25$ stents were implanted per patient. Bifurcation lesions were identified in $90.1 \%$ and the most frequent techniques were the provisional stent in $36 \%$ and small crush in $29 \%$ of the patients. Intravascular ultrasound was performed in $92.3 \%$ of the patients and reinterventions in $21.3 \%$ of the stents due to incomplete apposition of the struts after implantation. In the late follow-up, MACE was observed in $15.4 \%$ of the patients, cardiac death in $3.6 \%$, target-vessel revascularization in $11.2 \%$ and definitive/probably stent thrombosis in $1.4 \%$. Conclusions: In this study, $\mathrm{PCl}$ with DES proved to be safe and effective in the late follow-up of LM lesions, with low cardiac death and stent thrombosis rates.

KEY-WORDS: Coronary artery disease. Angioplasty. Drugeluting stents.

\footnotetext{
1 Hospital Cardiológico Costantini - Fundação Francisco Costantini Curitiba, PR, Brasil.

Correspondência: Costantino R. Costantini. Rua Pedro Colere, 890 Vila Izabel - Curitiba, PR, Brasil - CEP 80320-320

E-mail: crcostantini@hospitalcostantini.com.br

Recebido em: 5/4/2011 • Aceito em: 2/6/2011
} 
A s lesões de troco de coronária esquerda estão presentes em $5 \%$ a $9 \%$ dos pacientes portadores de aterosclerose coronária. ${ }^{1}$ A revascularização cirúrgica é considerada a primeira opção terapêutica para esses pacientes, quando comparada ao tratamento clínico. ${ }^{2}$ Introduzida em 1977, a intervenção coronária percutânea $(\mathrm{ICP})^{3}$ experimentou notável avanço tecnológico, o que tornou possível o tratamento de lesões coronárias cada vez mais complexas. ${ }^{4}$ Nesse período, o tratamento das lesões de tronco de coronária esquerda não-protegido (TCE-NP) tem sido avaliado. Nos primeiros ensaios com o uso de cateter-balão, os resultados iniciais foram desanimadores, ao demonstrar mortalidade relacionada ao procedimento de $9,1 \%$ em pacientes eletivos e de $50 \%$ em pacientes portadores de infarto agudo do miocárdio (IAM). ${ }^{5}$ Com a introdução dos stents não-farmacológicos (SNFs) observou-se melhora dos resultados, com maior previsibilidade dos resultados agudos, porém a incidência de reestenose limitava seu uso na prática diária. ${ }^{6} \mathrm{~A}$ disponibilidade dos stents farmacológicos $(\mathrm{SFs})$ trouxe resultados mais favoráveis, com redução da reestenose, comparativamente aos SNFs. ${ }^{7,8}$ Registros recentes demonstram que o implante de SFs de forma eletiva em pacientes selecionados com lesão de TCE-NP é uma alternativa viável.7,9

O objetivo deste estudo foi avaliar os resultados clínicos da ICP com SFs em pacientes com lesão de TCE-NP.

\section{MÉTODOS}

\section{Desenho do estudo e seleção dos pacientes}

Este estudo consiste de um registro prospectivo, unicêntrico e de braço único, que avaliou a segurança e a eficácia do uso de SFs em pacientes portadores de lesões de TCE-NP.

Entre março de 2002 e março de 2011, foram realizadas no Hospital Cardiológico Costantini (Curitiba, PR, Brasil), no total, 6.130 ICPs, sendo identificados 142 pacientes com lesão de TCE-NP tratados com SFs e acompanhados tardiamente. Desses pacientes, 103 completaram um ano de evolução e 75, dois anos de evolução. A decisão de realizar ICP foi considerada quando o paciente apresentava anatomia favorável e recusava o procedimento cirúrgico ou quando apresentava alto risco de mortalidade cirúrgica, avaliado pelo EuroSCORE. ${ }^{10}$

Todos os pacientes assinaram termo consentindo o procedimento.

\section{Procedimento de intervenção coronária}

O acesso arterial foi obtido por via femoral em todos os pacientes e os procedimentos foram realizados de acordo com a técnica padrão. ${ }^{11} \mathrm{~A}$ decisão de utilizar um ou dois stents e o uso adjunto de inibidor da glicoproteína Ilb/IIla (GP Ilb/IIla) ficou a critério de cada operador. A pré-dilatação poderia ou não ser realizada, mas a pós-dilatação era aplicada a todos os pacientes, independentemente do aspecto visual obtido pela angiografia. Heparina não-fracionada foi administrada na dose de $100 \mathrm{Ul} / \mathrm{kg}$, com o objetivo de obter tempo de coagulação ativada $>250$ segundos para aqueles pacientes que não fizeram uso de GP Ilb/IIla, e de $70 \mathrm{UI} / \mathrm{kg}$ para aqueles que fizeram uso adjunto de GP IIb/IIla. A terapia com dupla agregação antiplaquetária com ácido acetilsalicílico (dose de ataque de $300 \mathrm{mg} /$ dose de manutenção de $100 \mathrm{mg}$ ) e clopidogrel (dose de ataque de $300 \mathrm{mg} /$ dose de manutenção de $75 \mathrm{mg}$ ) era iniciada 24 horas antes do procedimento. Para os pacientes tratados com ticlopidina, a dose administrada era de $250 \mathrm{mg}$ a cada 12 horas, iniciada três dias antes do procedimento. O ácido acetilsalicílico era mantido indefinidamente e os tienopiridínicos eram mantidos por pelo menos 12 meses, se não houvesse complicações hemorrágicas.

\section{Ultrassom intracoronário}

Para a aquisição das imagens do ultrassom intracoronário (USIC) foi utilizado transdutor de elemento único, rotacional, com frequência de $40 \mathrm{MHz}$, envolto em bainha com perfil 2,6 F, com recuo motorizado, em sistema de tração automática à velocidade de $0,5 \mathrm{~mm} / \mathrm{s}$, conectado a um escâner comercialmente disponível (CVIS, Galaxy 2 e iLAB 2 - Boston Scientific Corporation, Natick, Estados Unidos). As imagens foram digitalizadas para análise quantitativa off-line de acordo com os critérios do American College of Cardiology's Clinical Expert Consensus Document on IVUS ${ }^{12}$, realizada com o programa EchoPlaque 3.0.48 (INDEC Systems Inc., Mountain View, Estados Unidos). As áreas de lúmen, stent e vaso (membrana elástica externa) no segmento analisado foram determinadas, a cada milímetro, por planimetria computadorizada. Na análise qualitativa, aposição incompleta do stent foi definida como a separação de pelo menos uma de suas hastes da parede vascular, não envolvendo uma área de bifurcação e a identificação de fluxo de sangue por trás das hastes. ${ }^{13}$

\section{Definições}

Todos os óbitos foram considerados de origem cardíaca, a menos que uma causa não-cardíaca fosse inequivocamente identificada. IAM não-fatal incluiu: IAM periprocedimento, definido em conformidade com as recomendações da força tarefa ESC/ACC/AHA/WHF ${ }^{14}$, como elevação dos níveis de troponina ou creatina quinase fração $M B$ (CK-MB) maior que três vezes o limite superior da normalidade até 48 horas após a ICP; e IAM espontâneo, definido conforme recomendação do Academic Research Consortium (ARC) ${ }^{15}$, como qualquer elevação de troponina ou CK-MB acima do valor superior de normalidade do laboratório, não relacionada com procedimentos de ICP, em qualquer momento após a intervenção índex. Revascularização do vaso-alvo (RVA) foi definida como a necessidade de revascularização 
percutânea ou cirúrgica no vaso previamente tratado ou, no caso de a lesão inicial incluir a bifurcação do TCE-NP, a revascularização da artéria descendente anterior ou artéria circunflexa. Trombose dos stents foi definida conforme as recomendações do ARC, sendo classificada de acordo com o nível de evidência (definitiva, provável ou possível) e com o tempo de ocorrência (aguda, subaguda, tardia e muito tardia). O sucesso do procedimento foi definido como a obtenção de estenose residual $<20 \%$, de acordo com a análise angiográfica e ultrassonográfica, sem a ocorrência de morte, IAM Q ou RVA percutânea ou cirúrgica. O escore SYNTAX, que se baseia nas características angiográficas, foi utilizado para graduar a complexidade das lesões coronárias.

Eventos clínicos combinados (morte, IAM, RVA ou trombose do stent) e isolados foram avaliados em todos os pacientes, com acompanhamento por atendimento ambulatorial ou entrevistas por telefone. A angiografia coronária de rotina durante o seguimento não foi realizada, sendo deixada a critério clínico, com base nos sintomas apresentados e/ou nos resultados de testes não-invasivos.

Na análise descritiva dos dados, as variáveis categóricas foram expressas como frequências absolutas e porcentuais e as variáveis contínuas, como média \pm desvio padrão.

\section{RESULTADOS}

No período de março de 2002 a março de 2011, 142 pacientes consecutivos com lesão de TCE-NP foram tratados com SFs, com média de seguimento de $917 \pm$ 743 dias. As características clínicas são apresentadas na Tabela 1. A média das idades da população foi de $67,5+16$ anos, $33 \%$ dos pacientes tinham mais de 75 anos, três quartos dos pacientes eram do sexo masculino, 29\% eram portadores de diabetes melito e 39\% dos pacientes apresentavam angina instável. Foram excluídos da análise 2 pacientes admitidos com diagnóstico de IAM.

Foi utilizado introdutor $7 \mathrm{~F}$ ou $8 \mathrm{~F}$, sendo o óstio da coronária esquerda alcançado com cateter XB $7 \mathrm{~F}$ em 59 pacientes $(42 \%)$, XB $8 \mathrm{~F}$ em 58 pacientes $(41 \%), \mathrm{FL}$ $7 \mathrm{~F}$ em 12 pacientes (8\%), FL $8 \mathrm{~F}$ em 9 pacientes $(6 \%)$, e Vista Brite XB $6 \mathrm{~F}$ em 4 pacientes (3\%). Apenas 4 pacientes $(2,8 \%)$ fizeram uso de balão intra-aórtico. $\mathrm{O}$ tratamento do TCE privilegiando a artéria descendente anterior foi a estratégia mais frequente $(63 \%)$. Foram utilizados 2,75+1,25 stents por paciente. Os SFs implantados foram 75 Taxus $^{\mathrm{TM}}$ (Boston Scientific, Natick, Estados Unidos), 27 Cypher $^{\mathrm{TM}}$ (Cordis, Miami, Estados Unidos), 14 Promus $^{\mathrm{TM}}$ (Boston Scientific, Natick, Estados Unidos), e 26 outros (Biomatrix ${ }^{\mathrm{TM}}$ - Biosensors International, Cingapura; Endeavor ${ }^{\mathrm{TM}}$ - Medtronic, Minneapolis, Estados Unidos; Supralimus ${ }^{\mathrm{TM}}$ - Sahajanand Medical Technologies Pvt. Ltd., Surat, Índia; Xience ${ }^{\mathrm{TM}}$
TABELA 1

Características clínicas basais

\begin{tabular}{|c|c|}
\hline & $n=142$ \\
\hline Idade, anos (média \pm DP) & $67,5 \pm 16$ \\
\hline Sexo masculino, n (\%) & $107(75)$ \\
\hline Hipertensão arterial, n (\%) & $119(84)$ \\
\hline Diabetes melito, n (\%) & $41(29)$ \\
\hline Dislipidemia, n (\%) & $113(80)$ \\
\hline Tabagismo atual, n (\%) & $32(23)$ \\
\hline Insuficiência renal crônica, n (\%) & $3(2)$ \\
\hline ICP prévia, n (\%) & $55(39)$ \\
\hline CRM prévia, n (\%) & $8(6)$ \\
\hline \multicolumn{2}{|l|}{ EuroSCORE, n (\%) } \\
\hline Baixo risco (0-2) & $16(11)$ \\
\hline Médio risco (3-5) & $48(34)$ \\
\hline Alto risco $(\geq 6)$ & $78(55)$ \\
\hline \multicolumn{2}{|l|}{ Diagnóstico clínico, n (\%) } \\
\hline Isquemia silenciosa & $24(18)$ \\
\hline Angina estável & $46(32)$ \\
\hline Angina instável & $56(39)$ \\
\hline Outros sintomas* & $16(11)$ \\
\hline \multicolumn{2}{|c|}{$\begin{array}{l}\text { * Angina atípica, equivalente anginoso. } \\
\text { CRM = cirurgia de revascularização miocárdica; DP = desvio } \\
\text { padrão; ICP = intervenção coronária percutânea; } \mathrm{n}=\text { número } \\
\text { de pacientes. }\end{array}$} \\
\hline
\end{tabular}

Abbott Laboratories, Abbot Park, Estados Unidos). As médias dos diâmetros e dos comprimentos dos stents implantados foram de 3,8 $\pm 0,5 \mathrm{~mm}$ e $26,1 \pm 8,4 \mathrm{~mm}$, respectivamente. Sucesso angiográfico foi obtido em todos os pacientes $(100 \%)$. Sucesso ultrassonográfico foi obtido em 112 pacientes (78,9\%), e os demais 30 pacientes $(21,3 \%)$ necessitaram reintervenção para a obtenção de sucesso ultrassonográfico.

\section{Angiografia coronária quantitativa}

A Tabela 2 apresenta os resultados angiográficos. Lesões com comprometimento da bifurcação foram identificadas em 112 pacientes pela angiografia, acrescidas de outras 16, após a avaliação ultrassonográfica, totalizando 128 lesões (90,1\%). Apresentavam doença multiarterial 123 pacientes (87\%), dos quais $63(51 \%)$ eram de alta complexidade (escore SYNTAX > 33), e 49 pacientes $(35 \%)$ tinham estenose significativa da artéria coronária direita associada. Tirofiban foi utilizado em 36 pacientes (25\%).

A Tabela 3 apresenta a técnica utilizada no procedimento e seus resultados imediatos. Pré-dilatação foi realizada em $86 \%$ das lesões, decisão tomada após análise ultrassonográfica ou a critério do operador. Insuflação com a técnica de kissing-balloon pré-implante do stent foi realizada em $24 \%$ e kissing-balloon no final do procedimento em $83 \%$ dos casos que receberam 
TABELA 2

Características angiográficas basais e do procedimento

\begin{tabular}{|c|c|}
\hline & $n=142$ \\
\hline \multicolumn{2}{|l|}{ Localização, n (\%) } \\
\hline Ostial/corpo & $30(21)$ \\
\hline Tipo de bifurcação* & $112(79)$ \\
\hline (1.0.0) & $4(4)$ \\
\hline$(0.1 .0)$ & $11(10)$ \\
\hline$(1.1 .0)$ & $7(6)$ \\
\hline$(1.1 .1)$ & $32(29)$ \\
\hline$(0.0 .1)$ & $14(13)$ \\
\hline$(1.0 .1)$ & $42(38)$ \\
\hline \multicolumn{2}{|l|}{ Escore SYNTAX, n (\%) } \\
\hline Baixo risco $(0-22)$ & $26(21)$ \\
\hline Médio risco (23-32) & $34(28)$ \\
\hline Alto risco $(\geq 33)$ & $63(51)$ \\
\hline Lesão em coronária direita, n (\%) & $49(35)$ \\
\hline Doença multiarterial, n (\%) & $123(87)$ \\
\hline Balão intra-aórtico, n (\%) & $4(3)$ \\
\hline $\begin{array}{l}\text { Uso de inibidores da } \\
\text { glicoproteína IIb/IIla, n (\%) }\end{array}$ & $36(25)$ \\
\hline ICP guiada por USIC & $131(92,3)$ \\
\hline
\end{tabular}

TABELA 3

Técnica utilizada e resultados do procedimento

\begin{tabular}{|c|c|}
\hline & $n=142$ \\
\hline Bifurcações tratadas, n (\%) & $128(89)$ \\
\hline Pré-dilatação, n (\%) & $122(86)$ \\
\hline \multicolumn{2}{|l|}{ Técnica utilizada, n (\%) } \\
\hline Provisional stent & $45(36)$ \\
\hline Small crush & $37(29)$ \\
\hline Small crush invertido & $16(13)$ \\
\hline T stent & $6(5)$ \\
\hline T stent invertido & $5(4)$ \\
\hline Crush & $5(4)$ \\
\hline Small crush moderado & $5(4)$ \\
\hline Crush invertido & $3(2)$ \\
\hline SKS & $3(2)$ \\
\hline Kissing-balloon pré-stent, n (\%) & $30(24)$ \\
\hline Kissing-balloon final, n (\%) & $105(83)$ \\
\hline Stents por paciente (média \pm DP) & $2,75 \pm 1,25$ \\
\hline Sucesso angiográfico, n (\%) & $142(100)$ \\
\hline Sucesso ultrassonográfico, n (\%) & $115(81)$ \\
\hline
\end{tabular}

DLM = diâmetro luminal mínimo; DP = desvio padrão; $\mathrm{n}=$ número de pacientes; SKS = simultaneous kissing stent. um ou dois stents. A média dos diâmetros de referência dos vasos tratados foi de 3,6 $\pm 0,6 \mathrm{~mm}$ para a angiografia quantitativa e de 4,1 +0,4 mm para a análise ultrassonográfica, com extensão média de 25,4 \pm 7,8 mm.

USIC foi realizado em 131 pacientes (92,3\%) e determinou a escolha de stent com maior diâmetro e comprimento, quando comparado com a análise pela angiografia coronária quantitativa. Reintervenção ocorreu em 30 stents $(21,3 \%)$, por apresentarem aposição incompleta de suas hastes imediatamente após o implante.

A Tabela 4 apresenta os resultados da angiografia coronária quantitativa e do ultrassom intracoronário.

\section{Resultado clínico hospitalar}

Durante a evolução hospitalar, um paciente apresentou trombose provável do stent e óbito. Esse paciente, tratado em 2004, apresentava lesão grave de óstio de TCE e lesão segmentar grave de artéria descendente anterior, foi submetido a aterectomia rotacional, que resultou em dissecção coronária, sendo necessário o implante de três stents (dois SNFs e um SF liberador de paclitaxel, no segmento proximal da artéria descendente anterior até o óstio do TCE). Ao término do procedimento encontrava-se estável clínica e hemodinamicamente e foi encaminhado para unidade de cuidados intensivos, onde, oito horas após, apresentou agitação psicomotora, fibrilação ventricular e óbito.

Outro paciente realizou ICP em 2010, portador de doença multiarterial, IAM prévio, EuroSCORE > 6 e escore SYNTAX > 33, com fração de ejeção de 34\%, necessitando do sistema Impella para suporte hemodinâmico durante o procedimento. Apresentou sucesso angiográfico e clínico inicial com implante de um SF liberador de paclitaxel, evoluindo com trombose aguda de stent dentro das 24 horas e IAM. Apesar de ter sido realizada RVA, o paciente evoluiu com choque cardiogênico e óbito. Nenhum paciente necessitou cirurgia de revascularização miocárdica (CRM) de urgência.

\section{Seguimento clínico tardio}

Dados acerca do seguimento clínico após o procedimento foram obtidos de todos os pacientes, por consulta ambulatorial ou contato telefônico. A Tabela 5 apresenta a taxa de ocorrência dos desfechos na totalidade de pacientes, em seguimento clínico médio de 917 + 743 dias. Desfechos primários ocorreram em 22 pacientes $(15,4 \%)$ e a mortalidade global por qualquer causa foi de $6,4 \%$, sendo $3,6 \%$ (5 pacientes) de causa cardíaca e $2,8 \%$ (4 pacientes) de causa não-cardíaca.

\section{DISCUSSÃO}

O principal achado deste estudo foi que em pacientes com lesão de TCE-NP, tratados eletivamente com SF e acompanhados a longo prazo (média de $917 \pm$ 743 dias), a incidência de eventos cardíacos adversos maiores foi de 15,4\%; de óbito cardíaco, 3,6\%; de RVA, $11,2 \%$; e de trombose definitiva/provável do stent, 1,4\%. 
TABELA 4

Angiografia coronária quantitativa e ultrassom intracoronário

\begin{tabular}{lcc}
\hline & $\begin{array}{c}\text { ACQ } \\
\text { (142 avaliações) }\end{array}$ & $\begin{array}{c}\text { USIC } \\
\text { (131 avaliações) }\end{array}$ \\
\hline Pré-procedimento & & \\
Comprimento do TCE, mm & $11,4 \pm 4,2$ & $11,9 \pm 1,2$ \\
Comprimento da lesão, mm & $5,5 \pm 3,3$ & $9,5 \pm 3,3$ \\
Diâmetro de referência, mm & $3,6 \pm 0,6$ & $4,1 \pm 0,4$ \\
DLM, mm² & $1,9 \pm 0,8$ & $1,9 \pm 0,1$ \\
Diâmetro da estenose, \% & $46,5 \pm 22,5$ & $65,6 \pm 11,1$ \\
Área da estenose, \% & $\mathrm{N} / \mathrm{A}$ & $67,1 \pm 7,5$ \\
Pós-procedimento & & \\
Diâmetro de referência, mm & $3,8 \pm 0,5$ & $4,2 \pm 0,5$ \\
DLM, mm & $3,4 \pm 0,6$ & $3,9 \pm 0,3$ \\
Diâmetro da estenose, \% & $11,4 \pm 8,5$ & $5,2 \pm 1,1$ \\
Área da estenose, \% & $\mathrm{N} / \mathrm{A}$ & $18,2 \pm 4,2$ \\
\hline
\end{tabular}

$\mathrm{ACQ}$ = angiografia coronária quantitativa; DLM = diâmetro luminal mínimo; N/A = não aplicável; TCE = tronco de coronária esquerda; USIC = ultrassom intracoronário.

TABELA 5

Desfechos clínicos iniciais e tardios

\begin{tabular}{lc}
\hline & $\mathbf{n}=\mathbf{1 4 2}$ \\
\hline Seguimento clínico, dias (média \pm DP) & $917 \pm 743$ \\
Período hospitalar, n (\%) & $2(1,4)$ \\
ECAM & $2(1,4)$ \\
Óbito cardíaco & $1(0,7)$ \\
IAM & $1(0,7)$ \\
RLA & \\
Período tardio, n (\%) & $22(15,4)$ \\
ECAM & $5(3,6)$ \\
Óbito cardíaco & $4(2,8)$ \\
Óbito não-cardíaco & $2(1,4)$ \\
Infarto do miocárdio & $16(11,2)$ \\
RVA & \\
Trombose definitiva/provável de stents & $2(1,4)$ \\
Aguda & 0 \\
Subaguda & 0 \\
Tardia & \\
\hline DP = desvio padrão; ECAM = eventos cardíacos adversos \\
maiores; IAM = infarto agudo do miocárdio; $\mathrm{n}=$ número de \\
pacientes; RLA = revascularização da lesão-alvo; RVA = \\
revascularização do vaso-alvo. \\
\hline
\end{tabular}

Diretrizes atuais de revascularização miocárdica ${ }^{16}$ trazem grau de recomendação Ila para intervenção com SF em lesões de corpo e óstio de TCE-NP e Ilb para lesões localizadas na bifurcação. Essas recomendações baseiam-se em estudos que demonstram a superioridade dos SFs comparativamente aos SNFs nesse cenário anatômico ${ }^{17}$ e nos resultados recentes dos estudos SYNTAX. ${ }^{18}$

O registro LE MANS ${ }^{17}$ avaliou os resultados iniciais e tardios da ICP no tratamento da lesão de TCE-NP, comparando SF e SNF. Incluiu 252 pacientes, 36,2\% tratados com SF. Eventos cardíacos e cerebrovasculares maiores (ECCM) ocorreram em 4,8\% dos pacientes aos 30 dias, incluindo mortalidade em 1,5\%. Em acompanhamento médio de 3,8 anos, ECCM ocorreram em $25,4 \%$ dos pacientes e mortalidade, em $13,9 \%$. Os SFs cursaram com menor ECCM que os SNFs (14,9\% vs. $25,9 \% ; P=0,039)$, diferença ampliada após pareamento para ajustar diferenças clínicas e angiográficas entre os grupos.

O estudo randomizado SYNTAX ${ }^{18}$, que avaliou CRM vs. ICP com implante de stents Taxus ${ }^{\mathrm{TM}}$ em pacientes com lesões em três vasos e TCE-NP, demonstrou que a ICP falhou em alcançar a meta de não-inferioridade comparada à CRM para os ECCM combinados, embora as duas estratégias tenham demonstrado taxas comparáveis de óbito, IAM, e acidente vascular cerebral ao final de um ano. O acidente vascular cerebral foi mais frequente com a CRM e a RVA, nos pacientes tratados com ICP. No subgrupo de pacientes com lesão de TCE$\mathrm{NP}^{19}$, quando os pacientes foram classificados pela complexidade anatômica, aqueles com escore SYNTAX baixo $(7,7 \%$ vs. $13 \%$; $P=0,19)$ ou intermediário $(12,6 \%$ vs. $15,5 \% ; P=0,54)$ apresentaram resultados clínicos equivalentes entre a ICP e a CRM; os pacientes com escore alto apresentaram menos eventos quando tratados cirurgicamente $(25,3 \%$ vs. $12,9 \% ; P=0,008)$.

O tratamento das lesões de óstio e do corpo do TCE-NP com SFs demonstra taxas de ECAM mais favo- 
ráveis. Em acompanhamento de $886 \pm 308$ dias, 147 pacientes tratados com stents com eluição de sirolimus ou paclitaxel demonstraram taxas de ECAM de 7,4\% e de reestenose de apenas $0,9 \% .^{20}$ Já as lesões de bifurcação evoluem com eventos adversos mais frequentes. Análise do subgrupo de 476 pacientes com lesão de TCE-NP do Registro j-Cypher demonstrou que as taxas de revascularização da lesão-alvo (RLA) foram maiores nas lesões que acometeram a bifurcação, comparadas àquelas do óstio e do corpo do TCE-NP $(17,1 \%$ vs. $3,6 \% ; P=0,005)$. Nas lesões de bifurcação desse registro, pacientes tratados com stents nos ramos principal e secundário $(\mathrm{n}=119)$ evoluíram com taxas de óbito cardíaco $(12,2 \%$ vs. 5,5\%; P = 0,02) e RLA (30,9\% vs. $11,1 \% ; P<0,0001)$ superiores às daqueles tratados com stent apenas no ramo principal $(n=261){ }^{21}$

A incidência de trombose tardia e muito tardia em pacientes tratados com SF de primeira geração é baixa. Em um grande registro multicêntrico com 731 pacientes, a trombose definitiva ou provável em seguimento de $29,5 \pm 13,7$ meses foi de $0,9 \% .^{22}$

Nesta casuística, a taxa de mortalidade hospitalar foi de $1,4 \%$, resultado semelhante ao observado na metanálise de Biondi-Zoccai et al. ${ }^{23}$, em que foram avaliados 16 estudos com um total de 1.278 pacientes, com mortalidade de 1,7\% em 30 dias. Os resultados tardios também comparam-se aos demonstrados na literatura, considerando que os pacientes incluídos neste registro eram, em sua maioria, portadores de doença multiarterial e a ICP envolveu bifurcações e escore SYNTAX $\geq 33$ em 44,3\% da população.

\section{Limitações do estudo}

A principal limitação do presente estudo é o tamanho da população incluída, que pode ser insuficiente para avaliar desfechos de segurança. No entanto, seu valor reside no fato de representar uma das primeiras experiências clínicas no Brasil com SFs em pacientes com lesão de TCE-NP e de alta complexidade clínica e angiográfica acompanhados a longo prazo. Deve-se mencionar também o fato de ser um registro de braço único, observacional e não-randomizado.

\section{CONCLUSÕES}

Na presente análise envolvendo pacientes da prática diária com doença aterosclerótica em TCE-NP, a ICP com SF mostrou ser segura e eficaz na evolução tardia, com baixas taxas de óbito cardíaco e trombose do stent.

\section{CONFLITO DE INTERESSES}

Os autores declaram não haver conflito de interesses relacionado a este manuscrito.

\section{REFERÊNCIAS}

1. Farinha JB, Kaplan MA, Harris CN, Dunne EF, Carlish RA, Kay JH, Brooks S. Disease of left main coronary artery: surgical treatment and long term follow-up in 267 patients. Am J Cardiol. 1978;42(1):124-8.

2. Caracciolo EA, Davis KB, Sopko G, George CK, Kaiser GC, Corley SD, et al. Comparison of surgical and medical group survival in patients with left main coronary artery disease: Longterm CASS experience. Circulation. 1995;91(9):2325-34.

3. Grüntzig A. Transluminal dilatation of coronary-artery stenosis. Lancet. 1978;1(8058):263.

4. Bittl JA. Advances in coronary angioplasty. N Engl J Med. 1996;335(17):1290-302.

5. O'Keefe JH Jr, Hartzler GO, Rutherford BD, McConahay DR, Johnson WL, Giorgi LV, et al. Left main coronary angioplasty: early and late results of 127 acute and elective procedures. Am J Cardiol. 1989;64(3):144-7.

6. Park SJ, Park SW, Hong MK, Lee CW, Lee JH, Kim J, et al. Long-term (three-year) outcomes after stenting of unprotected left main coronary artery stenosis in patients with normal left ventricular function. Am J Cardiol. 2003;91(1):12-6.

7. Chieffo A, Stankovic G, Bonizzoni E, Tsagalou E, lakovou I, Montorfano M, et al. Early and mid-term results of drugeluting stent implantation in unprotected left main. Circulation. 2005;111(6):791-5.

8. Park SJ, Kim YH, Lee BK, Lee SW, Lee CW, Hong MK, et al. Sirolimus-eluting stent implantation for unprotected left main coronary artery stenosis: comparison with bare metal stent implantation. J Am Coll Cardiol. 2005;45(3):351-6.

9. Valgimigli $M$, van Mieghem CAG, Ong ATL, Aoki J, Rodriguez Granillo GA, McFadden EP, et al. Short-and long-term clinical outcome after drug-eluting stent implantation for the percutaneous treatment of left main coronary artery disease: insights from the Rapamycin-Eluting and Taxus Stent Evaluated At Rotterdam Cardiology Hospital registries (RESEARCH and T-SEARCH). Circulation. 2005;111(11):1383-9.

10. Nashef SA, Roques F, Michel P, Gauducheau E, Lemeshow S, Salamon R. European system for cardiac operative risk evaluation (EuroSCORE). Eur J Cardiothorac Surg. 1999;16(1):9-13.

11. King SB $3^{\text {rd }}$, Smith SC Jr, Hirshfeld JW Jr, Jacobs AK, Morrison DA, Williams DO, et al. 2007 focused update of the ACC/ AHA/SCAI 2005 guideline update for percutaneous coronary intervention: a report of the American College of Cardiology/ American Heart Association Task Force on Practice Guidelines. J Am Coll Cardiol. 2008;51(2):172-209.

12. Mintz GS, Nissen SE, Anderson WD, Bailey SR, Erbel R, Fitzgerald PJ, et al. American College of Cardiology Clinical Expert Consensus Document on Standards for Acquisition, Measurement and Reporting of Intravascular Ultrasound Studies (IVUS). A report of the American College of Cardiology Task Force on Clinical Expert Consensus Documents. J Am Coll Cardiol. 2001;37(5):1478-92.

13. Cook S, Wenaweser $P$, Togni $M$, Billinger $M$, Morger $C$, Seiler $\mathrm{C}$, et al. Incomplete stent apposition and very late stent thrombosis after drug-eluting stent implantation. Circulation. 2007;115(18):2426-34.

14. Thygesen K, Alpert JS, White HD, Jaffe AS, Apple FS, Galvani $M$, et al.; Joint ESC/ACCF/AHA/WHF Task Force for the Redefinition of Myocardial Infarction. Universal definition of myocardial infarction. Circulation. 2007;116(22):2634-53.

15. Cutlip DE, Windecker S, Mehran R, Boam A, Cohen DJ, van Es GA, et al.; Academy Research Consortium. Clinical end points in coronary stent trials: a case for standardized definitions. Circulation. 2007;115(17):2344-51.

16. European Association for Percutaneous Cardiovascular Interventions; Wijns W, Kolh P, Danchin N, Di Mario C, Falk $\mathrm{V}$, Folliguet $\mathrm{T}$, et al. Guidelines on myocardial revascularization: The Task Force on Myocardial Revasculariza- 
tion of the European Society of Cardiology (ESC) and the European Association for Cardio-Thoracic Surgery (EACTS). Eur Heart J. 2010;31(20):2501-55.

17. Buszman PE, Buszman PP, Kiesz RS, Bochenek A, Trela B, Konkolewska $M$, et al. Early and long-term results of unprotected left main coronary artery stenting: the LE MANS (Left Main Coronary Artery Stenting) registry. J Am Coll Cardiol. 2009;54(16):1500-11.

18. Serruys $\mathrm{P}$, Morice MC, Kappetein AP, Colombo A, Holmes DR, Mack MJ, et al.; SYNTAX Investigators. Percutaneous coronary intervention versus coronary-artery bypass grafting for severe coronary artery disease. N Engl J Med. 2009; 360(10):961-72.

19. Morice MC, Serruys PW, Kappetein AP, Feldman TE, Ståhle $\mathrm{E}$, Colombo A, et al. Outcomes in patients with de novo left main disease treated with either percutaneous coronary intervention using paclitaxel-eluting stent or coronary artery bypass graft treatment in the Synergy Between Percutaneous Coronary Intervention with TAXUS and Cardiac Surgery (SYNTAX) trial. Circulation. 2010;121(24):2645-53.
20. Chieffo A, Park SJ, Valgimigli M, Kim YH, Daemen J, Sheiban I, et al. Favorable long-term outcome after drug-eluting stent implantation in nonbifurcation lesions that involve unprotected left main coronary artery: a multicenter registry. Circulation. 2007;116(2):158-62.

21. Toyofuko $M$, Kimura $T$, Morimoto $T$, Hayashi $Y$, Ueda $H$, Kawai K, et al. Three-year outcomes after sirolimus-eluting stent implantation for unprotected left main coronary artery disease: insights from the j-Cypher registry. Circulation. 2009; 120(19):1866-74.

22. Chieffo A, Park SJ, Meliga E, Sheiban I, Lee MS, Latib A, et al. Late and very late stent thrombosis following drugeluting stent implantation in unprotected left main coronary artery: a multicentre registry. Eur Heart J. 2008;29(17): 2108-15.

23. Biondi-Zoccai GG, Lotrionte M, Moretti C, Meliga E, Agostoni $P$, Valgimigli $M$, et al. A collaborative systematic review and meta-analysis on 1278 patients undergoing percutaneous drug-eluting stenting for unprotected left main coronary artery disease. Am Heart J. 2008;155(2):274-83. 